\title{
Effect of material characteristics of explosive on formation of collinear explosively formed projectile from warhead with double layer liners
}

\author{
Sunlang Lin ${ }^{1}$, Changxiao Zhao ${ }^{2}$, Fangyu Zhang ${ }^{3}$, Chong $\mathrm{Ji}^{4}$, Leiyao Zhang ${ }^{5}$, \\ Mingshou Zhong ${ }^{6}$ \\ College of Field Engineering, Army Engineering University of PLA, Nanjing, China \\ ${ }^{2}$ Corresponding author \\ E-mail:158243911@qq.com, ${ }^{2} 360349734 @ q q . c o m,{ }^{3} 459316513 @ q q . c o m,{ }^{4} 2468645816 @ q q . c o m$, \\ 52534763887@qq.com,6109125659@qq.com
}

Received 8 November 2021; received in revised form 3 December 2021; accepted 16 December 2021 DOI https://doi.org/10.21595/vp.2021.22284

Check for updates

Copyright (C) 2022 Sunlang Lin, et al. This is an open access article distributed under the Creative Commons Attribution License, which permits unrestricted use, distribution, and reproduction in any medium, provided the original work is properly cited.

\begin{abstract}
In order to obtain the effect of material characteristics of explosive on the formation of collinear explosively formed projectile (EFP)from warhead with dosuble liners, the LS-DYNA simulation software was used to simulate and analyze the forming process of collinear EFP from warhead with five explosive materials including pe4booster, PETN, TNT, C4 and B explosives. The results indicate that with the increase of density, detonation velocity, and detonation pressure, the velocity and the length diameter ratio of the front EFP and rear EFP increase with different laws. With the increase of explosive material density, the velocity and the length diameter ratio of EFPs increase gradually, but the increasing rate decreases gradually. As the detonation velocity increases, the velocity of EFPs increase gradually, but the increasing rate decreases gradually. The length diameter ratio of EFPs decreases first and then increases gradually, showing an "s"-shaped change law. With the increase of explosive detonation pressure, the velocity of EFPs increase gradually, but the increasing rate decrease gradually. The length diameter ratio of the front EFP shows a parabolic growth law with the increase of detonation pressure, and the length diameter ratio of the rear EFP shows an "s"-shaped change law with the detonation pressure.
\end{abstract}

Keywords: mechanics of explosion, explosively formed projectile, double layer liners, explosive material.

\section{Introduction}

The collinear EFP from warhead with multi-layer liners can form multiple collinear EFPs separated from the front and rear EFPs. The front EFP is used to detonate the explosive reactive armor, and the rear EFP continues to penetrate the main armor, so as to effectively improve the damage efficiency to new armor. Therefore, it has become a hotspot of warhead technology research [1-6]. Faibish et al. [1] carried out numerical simulation and experimental research on the process of jet formation from the double-layer shaped charge with small cone angle copper liner on the outside and small cone angle tantalum liner on the insider. Ma et al. [2] obtained the specific process of the formation and separation of EFP with double layer liners by combining experiment and simulation. Tosello et al. [3] carried out numerical simulation and experimental research on the underwater motion of double-layer spherical defect hood composed of tantalum and nickel. Wang et al. [4] studied a double-layer liner with a copper inner cover and PTFE / Al reactive material outer cover and carried out the penetration experiment of the steel target.

According to the research of Zakiret al. [5], the change of explosive material used in charge will directly change the detonation energy, change the crushing forming process of the liner, and then affect the EFP forming parameters. However, there are few studies on the influence of explosive material on the formation of collinear EFP from warhead with double layer liners. Based on this, to analyze the influence of explosive materials on the collinear EFP forming, the process of the collinear EFP from warhead with double layer liners forming under different explosive 
materials was simulated by using LS-DYNA software, to obtain the variation law of EFP forming parameters of the coaxial double-layer liner with explosive material parameters, to provide a reference for warhead engineering design.

\section{Numerical simulation calculation and analysis}

\subsection{Numerical simulation calculation model}

The finite element model of EFP with double layer liners was established by using LS-DYNA software. The diameter and height of warhead are $10 \mathrm{~cm}$, and the double layer liners structure is adopted; The liners are all spherical shaped liners, in which the inner liner is close to the explosive and the outer liner is far away from the explosive; The thickness of the inner and outer liners is $0.5 \mathrm{~cm}$, and the arc radius of the inner circle is $26 \mathrm{~cm}$.

Because of the axisymmetric characteristics of the projectile and target structure and load, a $1 / 2$ model was established to save calculation time. Using the Lagrange algorithm, the specific calculation model is shown in Fig. 1. The liner is made of red copper and its density is $8.96 \mathrm{~g} / \mathrm{cm}^{3}$. In the calculation, the sliding penalty function algorithm is used between the explosive and the inner liner; Erosion contact is adopted between the inner and outer liner; A single liner defines automatic single-sided contact to simulate the contact of its outer surface after the liner is overturned, which can effectively avoid penetration and deformation between grids.

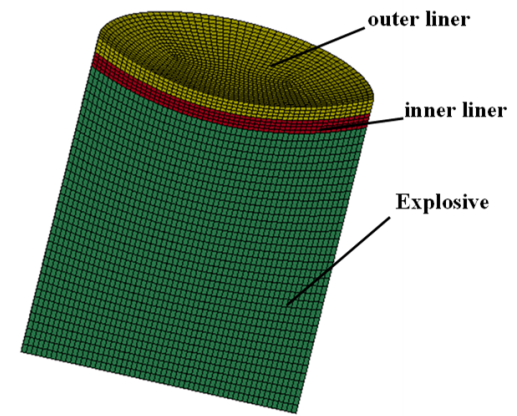

Fig. 1. The finite element model of EFP with double layer liners

\subsection{Analysis of simulation calculation results}

To find out the influence law of explosive material characteristics on the formation of damage element of double-layer EFP, five explosive materials are selected for simulation research: pe4booster, PETN, TNT, C4, and B explosives, to find out the influence law of explosive density, detonation velocity and detonation pressure on the formation parameters of the projectile. The main parameters of the five explosive material models are shown in Table 1.

Table 1. Explosive material parameters

\begin{tabular}{|c|c|c|c|c|c|c|c|c|c|}
\hline Material & $\rho_{c} / \mathrm{g} \cdot \mathrm{cm}^{-3}$ & $D_{w} / \mathrm{m}^{-} \mathrm{s}^{-1}$ & $A / \mathrm{GPa}^{-1}$ & $B / \mathrm{GPa}^{-1}$ & $R_{1}$ & $R_{2}$ & $\omega$ & $E / \mathrm{GPa}$ & $P / \mathrm{GPa}$ \\
\hline PETN & 1.260 & 6540 & 573.1 & 20.16 & 6 & 1.8 & 0.28 & 7.19 & 14 \\
\hline TNT & 1.460 & 7280 & 373.8 & 3.747 & 4.15 & 0.9 & 0.35 & 9.6 & 21 \\
\hline C4 & 1.601 & 8193 & 609.9 & 12.95 & 4.5 & 1.4 & 0.25 & 9.0 & 28.0 \\
\hline B & 1.717 & 7980 & 524.2 & 7.678 & 4.2 & 1.1 & 0.34 & 8.5 & 29.5 \\
\hline PE4BOOSTER & 1.590 & 7650 & 774.0 & 86.77 & 4.8 & 1.4 & 0.32 & 1.2 & 24.0 \\
\hline
\end{tabular}

The forming effect diagram of collinear EFP from warhead with double layer liners formed by different explosive materials is obtained by simulation calculation, as shown in Fig. 2, and the forming parameters are shown in Table 2. Among them, $L_{1}, R_{1}, v_{1}$ are the length, radius, speed and length diameter ratio of the rear EFP respectively, and $L_{2}, R_{2}, v_{2}$ are the length, radius, speed 
and length diameter ratio of the front EFP respectively.

It can be seen from Fig. 2 that there is a large difference in the shape of EFP formed by the collapse of the liner with different explosive materials, which mainly has a great impact on the length diameter ratio of the projectile, and the impact on the rear EFP is greater than the front EFP. In addition, it can be seen from Fig. 2 that the explosive detonation velocity has a significant impact on the forming parameters of EFP. With the increase of detonation velocity, the EFP formed by the inner liner and the outer liner gradually develops into a slender shape. Among them, the length diameter ratio of the projectile formed by explosive B is the largest.

The main parameters of explosive material such as density, detonation velocity, detonation pressure, and detonation heat are important factors affecting the forming of double-layer EFP. Therefore, to obtain the influence law of explosive material parameters on forming parameters of collinear EFP from warhead with double layer liners, numerical simulation methods are used below to study the influence law of explosive material density, detonation velocity, and detonation pressure on the forming parameters such as velocity and length diameter ratio of collinear EFP from warhead with double layer liners.

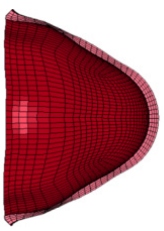

a) PETN

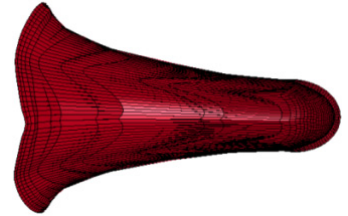

d) $\mathrm{B}$

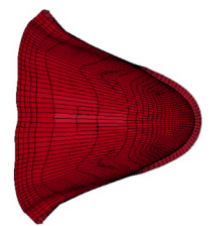

b) TNT

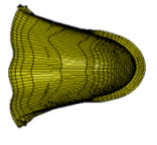

Fig. 2. Flying attitude of the projectile with different explosive materials

\subsubsection{Effect of explosive density}

According to the law of momentum conservation and the theory of instantaneous detonation, the EFP velocity can be deduced:

$v=\frac{\rho_{e} D}{\rho \sqrt{k^{2}-1}} S(x)$

where, $\rho$ is the material density of the liner, $k$ is the isentropic index of the explosive, $\rho_{e}$ is the explosive density, $D$ is the detonation velocity, and $S(x)$ is the integral function of the liner geometry.

According to Eq. (1), explosive density and detonation velocity is the direct factors affecting projectile forming. With the increase of density and detonation velocity, the EFP velocity will increase. By analyzing the parameters of double-layer EFP obtained by numerical simulation, the curve of velocity and length diameter ratio of collinear EFP from warhead with double layer liners with explosive density are obtained, as shown in Fig. 3. It can be seen from Fig. 3 that the explosive density has an important impact on the forming parameters of collinear EFP from warhead with double layer liners. With the increase of explosive density, the speed and length diameter ratio of the front and rear EFP increase. When the density increased from $1.26 \mathrm{~g} / \mathrm{cm}^{3}$ to $1.71 \mathrm{~g} / \mathrm{cm}^{3}$, the speed of the rear EFP increased by $33.7 \%$ and the length diameter ratio increased by $68.4 \%$; The speed of the front EFP increased by $41.3 \%$, and the length diameter ratio increased 
by $103.4 \%$. The effect of explosive density on the rear EFP than the front EFP. At the same time, it can be seen from Fig. 3 that the explosive density always has a positive proportional relationship with the forming parameters of double-layer EFP. The greater the density of explosive material, the more favorable it is for the forming of double-layer EFP, and the better it can improve the penetration ability to armored targets.

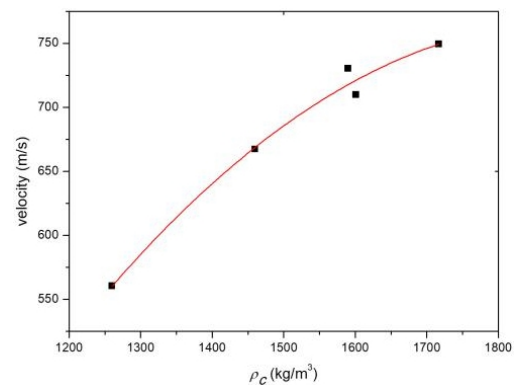

a) Speed of rear EFP

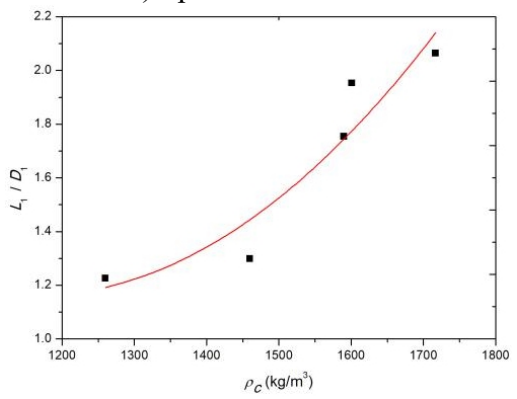

c) Length diameter ratio of rear EFP

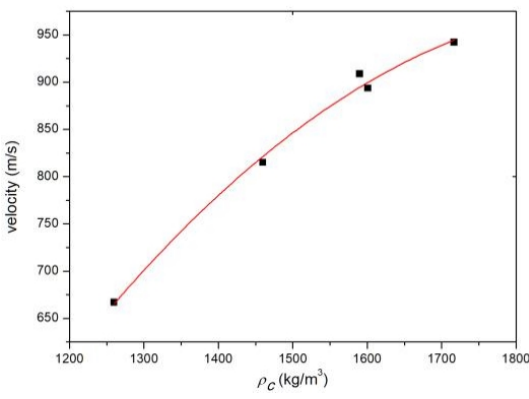

b) Speed of front EFP

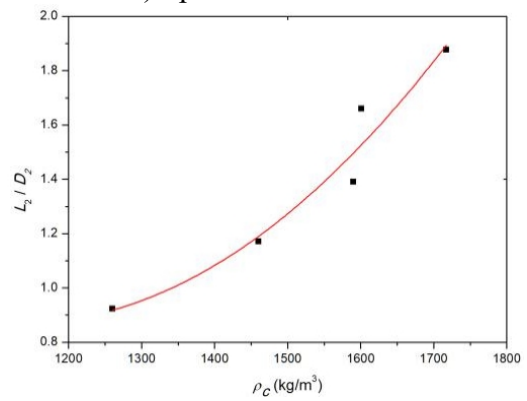

d) Length diameter ratio of front EFP

Fig. 3. Forming parameters of collinear EFP from warhead with double layer liners with explosive density

\subsubsection{Effect of detonation velocity}

Fig. 4 is the curve of forming parameters of collinear EFP from warhead with double liners with explosive detonation velocity. It can be seen from Fig. 4 that with the increase of detonation velocity, velocity and length diameter ratio of the front and rear EFP both decrease first, then increase and then decrease. When the detonation velocity increases from $6540 \mathrm{~m} / \mathrm{s}$ to $6900 \mathrm{~m} / \mathrm{s}$, the projectile velocity, and length diameter ratio change little. But it shows a linear upward trend when the detonation velocity continues to increase. When the detonation velocity increases to $7650 \mathrm{~m} / \mathrm{s}$, the upward trend of projectile forming parameters weakens. When the detonation velocity is greater than $7800 \mathrm{~m} / \mathrm{s}$, the EFP velocity and length diameter ratio of the front and rear stages both show a downward trend, and there is an optimal value for the explosive detonation velocity.

\subsubsection{Effect of detonation pressure}

Detonation pressure is one of the important indexes to measure the detonation energy, which directly affects the shape of the projectile formed by the liner deformation, thus affecting the final armor penetration effect. Therefore, by analyzing the influence of the detonation pressure of different explosives on the collinear EFP forming from warhead with double liners, the curve of forming parameters of collinear EFP from warhead with double liners with explosive detonation pressure is obtained, as shown in Fig. 5. With the increase of explosive detonation pressure, the speed and aspect ratio of front and rear EFP gradually increase. 


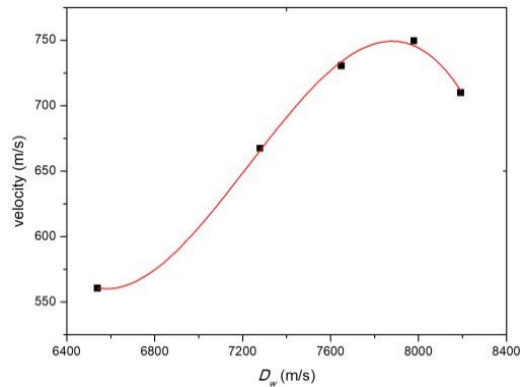

a) Speed of rear EFP

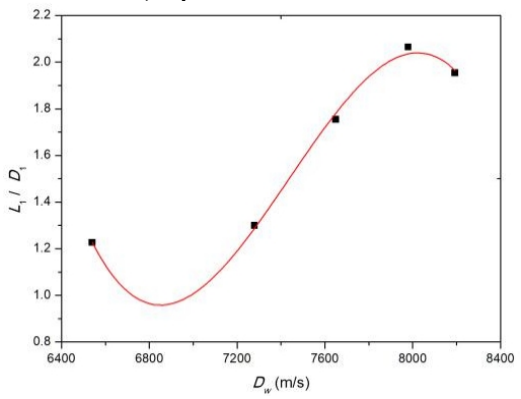

c) Length diameter ratio of rear EFP

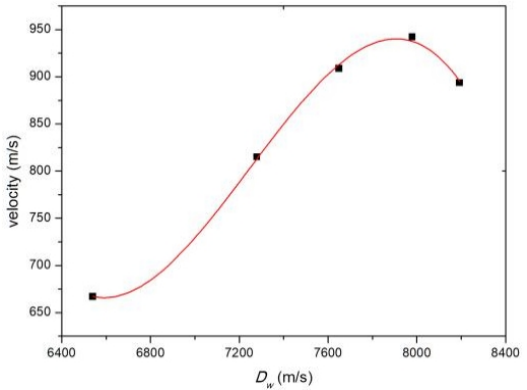

b) Speed of front EFP

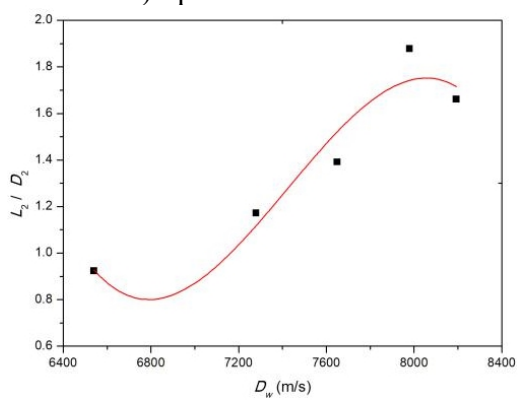

d) Length diameter ratio of front EFP

Fig. 4. Forming parameters of collinear EFP from warhead with double liners with explosive detonation velocity

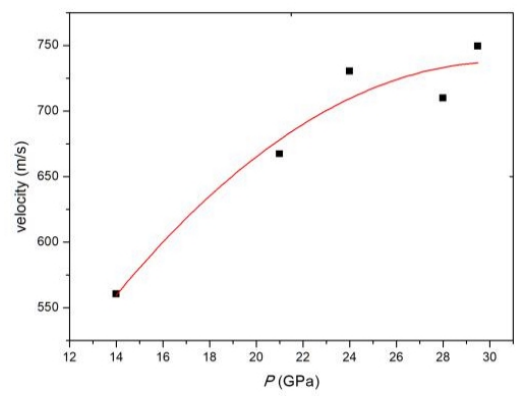

a) Speed of rear EFP

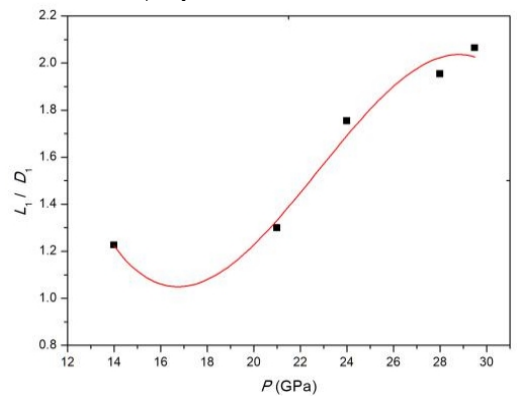

c) Length diameter ratio of rear EFP

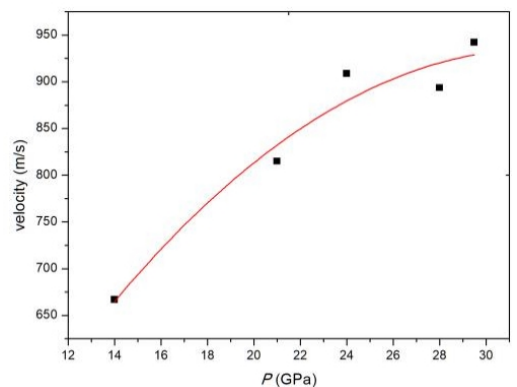

b) Speed of front EFP

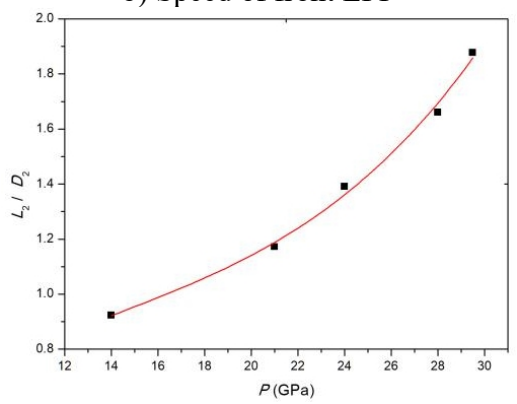

d) Length diameter ratio of front EFP

Fig. 5. Forming parameters of collinear EFP from warhead with double liners with explosive detonation pressure

When the detonation pressure increases from $14 \mathrm{GPa}$ to $29.5 \mathrm{GPa}$, the speed of the inner liner increases by $33.7 \%$, and the length diameter ratio increases by $68.4 \%$; The speed of the outer liner increases by $41.3 \%$, and the length diameter ratio increases by $103.4 \%$. The detonation 
pressure has a positive proportional relationship with the forming parameters of the double-layer EFP, but the length diameter ratio of the rear stage EFP presents an "s"-shaped change law with the detonation pressure. Generally speaking, increasing the detonation pressure can effectively improve the speed and length diameter ratio of the double-layer EFP and improve its damage ability.

\section{Conclusions}

In this paper, the effects of pe4booster, PETN, TNT, C4, and B explosives on forming parameters of collinear EFP from warhead with double layer liners are studied by simulation, and the law of the characteristic parameters such as explosive material density, detonation velocity, and detonation pressure and the forming parameters such as velocity and shape of the front and rear EFP are obtained. The results indicate that with the increase of density, detonation velocity, and detonation pressure, the velocity and the length diameter ratio of the front and rear EFP from warhead with double layer liners increase with different laws.

1) With the increase of explosive material density, the EFP velocity increases gradually, but the increasing rate decreases gradually, the length diameter ratio increases, and the increasing rate increases gradually.

2) As the detonation velocity increases, the EFP velocity increases gradually, but the increasing rate decreases gradually. The length diameter ratio of EFP decreases first and then increases gradually, showing an "s"-shaped change law.

3) With the increase of explosive detonation pressure, EFP velocity increases gradually, but the increasing rate decreases gradually. The length diameter ratio of the first EFP shows a parabolic growth law with the increase of detonation pressure, and the length diameter ratio of the rear EFP shows an "s"-shaped change law with the detonation pressure.

\section{Acknowledgements}

This research was financially supported by the National Nature Science Foundation of China, Nos. 12102479.

\section{References}

[1] Faibish E. and Mayseless M., "Double-layered conical liner manufactured by explosive welding," in Proceedings of the15th International Symposium on Ballistics, 1995.

[2] H.-B. Ma, Y.-F. Zheng, H.-F. Wang, C. Ge, and C.-H. Su, "Formation and impact-induced separation of tandem EFPs," Defence Technology, Vol. 16, No. 3, pp. 668-677, Jun. 2020, https://doi.org/10.1016/j.dt.2019.09.003

[3] Osello R., Vives M., and Tronche A., "Twin EFPs for underwater applications," in Proceedings of the18th International Symposium on Ballistics, 1999.

[4] H. Wang, H. Guo, B. Geng, Q. Yu, and Y. Zheng, "Application of PTFE/Al reactive materials for double-layered liner shaped charge," Materials, Vol. 12, No. 17, p. 2768, Aug. 2019, https://doi.org/10.3390/ma12172768

[5] "Numerical study on the optimum design of explosively formed projectile," in Proceedings of the 15th International Bhurban Conference on Applied Sciences and Technology, 2018.

[6] Dong Liying et al., "Anti-tank mine two way EFP forming and penetration simulation," (in Chinese), Journal of Ordnance Equipment Engineering, Vol. 40, No. 10, pp. 40-44, 2019. 